
allemande

47-2 | 2015

Retour sur le modèle Rhénan : humanisme, capitalisme et métropolisation | Patrimonialisation du passé « allemand » en Europe centrale après 1990

\title{
Muséaliser le passé des Allemands en Bohême : enjeux de représentation géographique et sociétale
}

Paul Bauer

\section{(QpenEdition \\ Journals}

Édition électronique

URL : https://journals.openedition.org/allemagne/307

DOI : 10.4000/allemagne.307

ISSN : 2605-7913

Éditeur

Société d'études allemandes

Édition imprimée

Date de publication : 16 décembre 2015

Pagination : 423-437

ISSN : 0035-0974

Référence électronique

Paul Bauer, « Muséaliser le passé des Allemands en Bohême : enjeux de représentation géographique et sociétale », Revue d'Allemagne et des pays de langue allemande [En ligne], 47-2 | 2015, mis en ligne le 13 décembre 2017, consulté le 19 mai 2021. URL : http://journals.openedition.org/allemagne/307 DOl : https://doi.org/10.4000/allemagne.307 


\section{Muséaliser le passé des Allemands en Bohême: enjeux de représentation géographique et sociétale}

\section{- Paul Bauer*}

La gestion du passé des Allemands de Tchécoslovaquie, dont la quasi-totalité a été déplacée de force vers l'Allemagne de l'Ouest et l'Autriche à la suite des accords de Potsdam en 1945, suit une trajectoire singulière depuis l'intégration de la République tchèque à l'Union européenne en 2004. Durant les quinze années suivant la chute des régimes socialistes en Europe centrale, la " question sudéto-allemande " ${ }^{(1)}$ a été au centre des crispations que connaissaient les relations bilatérales entre l'Allemagne réunifiée et la Tchécoslovaquie, devenue République tchèque et Slovaquie en 1993. Aujourd'hui, le passé des Allemands des pays tchèques est l'objet d'une attention nouvelle symbolisée par l'ouverture prochaine de l'« Exposition permanente de l'histoire et de la culture des habitants germanophones des pays tchèques " (Stalá exposice dějin a kultury německy mluvících obyvatel českých zemí) au sein du musée municipal d'Ústí nad Labem, au nord-est de la Bohême. Cette exposition a pour objectif de présenter les régions frontalières et les « îlots linguistiques » où vivait la majorité des Allemands des pays tchèques. L'exposition met en scène la présence des Allemands en Tchécoslovaquie, les siècles de cohabitation germano-tchèque, l'incorporation en 1938 des régions à majorité allemande dans le territoire du III ${ }^{e}$ Reich, le déplacement forcé de ces populations en 1945 et la gestion socialiste du passé dans les espaces qui ont subi des migrations forcées de population et le repeuplement d'après-guerre.

Pour le socio-géographe, l'intérêt de ce processus de muséalisation tient au fait que le passé allemand a été l'objet de politiques d'effacement et de " renationalisation » de la topographie et de la toponymie, auxquelles s'est ajoutée une refonte de la structure et de la morphologie des territoires repeuplés par une population, pour la très grande majorité,

* Docteur en géographie, chercheur à Université Charles de Prague, chercheur associé au CERCEC.

1 Michal Kopeček, Miroslav Kunštát, "Die Zwangsumsiedlung der Sudetendeutschen als Thema der tschechischen akademischen Debatte », in: Bernd Faulenbach \& Franz-Josef Jelich (dir.), 'Transformationen' der Erinnerungskulturen in Europa nach 1989, Essen, Klartext Verlag, 2006, p. 139-164; Christian JACQUES, «Les usages politiques de la mémoire "sudète” ", Revue d'Allemagne, 37/2 (2005), p. 218. 
étrangère à l'histoire des lieux ${ }^{(2)}$. D'un point de vue historiographique, l'exposition entend s'inscrire dans le débat sur " le sens de l'histoire tchèque » - une réflexion philosophique et politique d'inspiration hégélienne qui interroge l'universalité de l'histoire de la nation tchèque et sa place dans l'histoire du progrès européen ${ }^{(3)}$. Dans l'historiographie nationale tchèque des $\mathrm{XIX}^{\mathrm{e}}$ et $\mathrm{XX}^{\mathrm{e}}$ siècles, cette réflexion a été structurée par les approches scientifiques qui ont encadré le développement du nationalisme allemand (historicisme hégélien, théorie de la nation linguistique de Herder). Le mouvement national tchèque, qui s'est exprimé au cours du XIX siècle et dont est issu le débat sur "le sens de l'histoire tchèque ", s'est construit en opposition au mouvement national allemand dans un rapport de force dont l'historien tchèque Jan Křen a exposé l'histoire - à la fin des années 1980 dans un écrit samizdat - à partir du concept de "sociétés conflictuelles ${ }^{(4)}$.

Après 1989, le « sens de l'histoire tchèque » a été examiné par la communauté historienne à nouveau frais. L'introduction du socialisme d'État comme objet de réflexion avait en effet donné lieu à une remise en perspective des périodes antérieures. Anne Bazin rappelait au début des années 2000 que l'établissement d'une réflexion nouvelle sur le passé germano-tchèque constituait l'un des principaux moyens de la relecture de l'expérience du socialisme d'État - le retour de la «question allemande » étant lié en République tchèque à une réflexion sur les origines du totalitarisme ${ }^{(5)}$ - et les migrations forcées de population considérées comme la première rupture avec les principes démocratiques de la Première République tchécoslovaque (1918-1938).

Les transformations sociétales et territoriales consécutives aux déplacements forcés et leur lien au sens de l'histoire nationale tchèque forment le contexte socio-historique de ces réflexions et montrent que la géographie est au centre de la gestion du passé allemand dans les pays tchèques. Comment la géographie et la gestion du passé allemand s'articulent-elles dans le travail de muséalisation mis en œuvre à Ústí nad Labem? Le rapport au passé se construit-il en référence à l'espace et aux lieux? En quoi la réactualisation du passé des Allemands dans le projet d'exposition est-elle l'expression d'un projet sociétal postsocialiste inscrit dans la perspective du débat sur « le sens de l'histoire tchèque » et sur l'évaluation de la période socialiste?

Pour apporter des éléments de réponse à ces questions, cet article expose dans un premier temps les énoncés des discours sur la présence passée des Allemands tchèques. Il présente ensuite le contexte muséal du projet d'exposition et les cadres de connaissances dans lequel il s'inscrit ${ }^{(6)}$.

2 Adrian von Arburg, Tomaš StanĕK (dir.), Vysídlení Němců a proměny českého pohraniči 1945-1951: Dokumenty $z$ českých archivů (Le transfert des Allemands et les transformations des espaces frontaliers tchèques 1945-1951, documents des archives tchèques), díl. I a II., SUSA, 2010.

3 Voir à ce sujet, les deux recueils de textes de philosophes, d'historiens et de publicistes tchèques: Miloš Havelka (dir.), Spor o smysl českých dějin 1895-1938, Prague, TORST, 1997; Miloš Havelka (dir.), Spor o smysl českých dějin, 1938-1989, Prague, TORST, 2006.

4 Jan KřEn, Die Konfliktgemeinschaft: Tschechen und Deutsche 1780-1918, Munich, Oldenbourg, 2000.

5 Anne BAZIN, Les relations tchéco-allemandes depuis 1989, de la réconciliation bilatérale à l'intégration européenne, thèse de doctorat, IEP Paris, 2002, p. 446-447.

6 Les enquêtes de terrain dont cet article présente les résultats ont été réalisées dans le cadre du projet de recherche. "Space and Social Memory in the Czech Border Regions after 1990: The Post-socialist 


\section{Ignorance et déracinement: les énoncés du rapport au passé allemand en République tchèque}

Dans un entretien donné à un quotidien tchèque, Blanka Mouralová, directrice du centre de recherche chargé de réaliser l'exposition sur l'histoire de la culture des Allemands tchèques (le Collegium Bohemicum) s'exprime en ces termes: «La fin catastrophique des Allemands tchèques est la seule chose que nous connaissions. [...] Nous ne connaissons que très peu de choses des huit siècles qui font l'histoire germano-tchèque de la Bohême. [...] L’exposition permanente des Tchécoslovaques de langue allemande s'efforcera de présenter la manière dont les Allemands ont modelé les paysages et l'architecture actuels des pays tchèques ${ }^{(7)}$. Afin de légitimer la collection des artefacts qui seront présentés dans la future exposition, la directrice du Collegium Bohemicum met en avant l'ignorance de la société tchèque vis-à-vis de l'histoire de la présence allemande dans les pays tchèques. Ce rapport social à l'histoire des lieux, auquel Madame Mouralová fait référence, s'expliquerait par différents facteurs d'ordres socio-démographiques, territoriaux et politiques.

En premier lieu figure la volonté des autorités tchécoslovaques de faire émerger, à la suite des déplacements sur les lieux quittés par les Allemands, une société nationale tchèque composée de populations d'origines différentes arrivées au grès des vagues successives de repeuplement ${ }^{(8)}$. Aux raisons d'ordre démographique s'ajoutent des facteurs territoriaux: transformation de la structure et de la morphologie des espaces frontaliers, effacement progressif des traces de la présence allemande: effacement des noms des rues et des enseignes, abandon ou destruction des cimetières et des espaces sacrés dont l'utilisation était associée durant les premières années de la période socialiste à la vie des anciens habitants. Enfin, les spécialistes insistent sur la mise en place, dans l'après-guerre et durant toute la période socialiste, de dispositifs de contrôle de l'accès aux sources et de leur mise en scène dans les musées et les centres culturels et universitaires des espaces frontaliers. Dans les années qui suivirent le transfert forcé des populations allemandes et le repeuplement des zones abandonnées, le discours politique dominant présente la nouvelle ère qui s'ouvre à la "nation tchécoslovaque libérée » comme « l’ère des bâtisseurs » (Budovatelská éra $)^{(9)}$, consacrée à la reconstruction des espaces vidés et détruits. Durant ces années, les narrations sur le caractère

Handling of Material and Intangible Traces of the German Past ", financé par la Fondation tchèque pour la science (GAČR-P404/12/P297) et hébergé à l'Institut d'études sociologiques de la Faculté des sciences sociales de l'Université Charles à Prague.

7 «Češi a Němci se znají málo a nevěří si. To chci změnit » (Les Tchèques et les Allemands se connaissent peu et ne se font pas confiance. Je veux changer cela), MF Dnes, 1.06.2011.

8 Zdeněk Radvanoský, "Resettling Czechs into North Western Bohemia », in: Philippe Ther \& Ana SilJA K (dir.), Redrawing Nations: Ethnic Cleansing in East-Central Europe, 1944-1948, Boulder (Cold War Book Series, $\left.n^{\circ} 4\right), 2001$, p. 241-259.

9 Adrian von Arburg, "Konstruktéři nového pohraničí. Aktéři sídelní politiky a naplňování jejich vizí » (Les constructeurs des nouveaux espaces frontaliers. Les acteurs de la politique de repeuplement et la réalisation de leurs objectifs), Déjiny a současnost, XXXII/7, 2010, p. 14-17. Voir également du même auteur, "Peripherie oder Pionierland? Konzeptionen zur neuen Funktion des tschechischen Grenzgebiets 1945-1951 », in: Petr Lozoviuk (dir.), Grenzgebiet als Forschungsfeld: Aspekte der ethnografischen und kulturhistorischen Erforschung des Grenzlandes, Leipziger Universitätsverlag (Schriften zur sächsischen Geschichte und Volkskunde, 29), 2009, p. 85-112. 
national tchèque des espaces vidés s'imposent et fondent dans un contexte nouveau la relation entre la nation et le sol $^{(10)}$.

Ce n'est qu'au début des années 2000 que les discours sur le déracinement et l'ignorance des sociétés locales formées dans les villes et les villages anciennement peuplés d'Allemands apparaissent dans les colonnes des journaux, dans les analyses des spécialistes ainsi que dans les discours des acteurs politiques et associatifs locaux. Parmi ceux qui diffusent ces énoncés normatifs, l'association Antikomplex tient une place centrale qu'il faut expliciter.

Créée à la fin des années 1990 par des étudiants et des jeunes chercheurs (historiens, juristes, anthropologues) de l'Université Charles, proche de l'Académie chrétienne tchèque (Česká křestanská akademie) du prêtre tchèque Tomáš Halík, elle se fixe pour objectif de modifier les représentations de la société tchèque à l'égard de la présence passée des Allemands et de rompre avec les représentations sur les transferts forcés d'après-guerre héritées, selon eux, de la période du socialisme d'État ${ }^{(11)}$. Après quinze années d'existence, l'association s'est imposée comme l'un des principaux acteurs du débat portant sur le rapport des Tchèques à la présence passée de leurs compatriotes de langue allemande, grâce à un intense travail de sensibilisation aux conséquences sociaux-culturelles des transferts de population réalisé au moyen d'expositions itinérantes, de publications et de séminaires ${ }^{(12)}$. L'ancien président d'Antikomplex, Ondřej Matějka, a été nommé en 2014 vice-directeur de l'Institut pour l'étude des régimes totalitaires (Ústav pro studium totalitních režimů) dont les recherches sont centrées sur la période du Protectorat (1938-1945) et du socialisme d'État (1948-1989). Tandis que l'un des membres fondateurs est également le président de la Société Bernard Bolzano (du nom du célèbre mathématicien et philosophe tchèque, 1781-1848) qui rassemble des personnalités politiques de tout bord, des intellectuels et des membres des clergés catholiques et évangéliques afin de promouvoir l'idée d'une identité nationale fondée non pas sur la langue et le sang mais sur l'attachement à un territoire juridico-politique, sur un patriotisme territorial (zemský patriotismus) ${ }^{(13)}$.

L'association Antikomplex a très largement diffusé en République tchèque l'idée de l'importance sociétale de la relation morale des individus à leur espace de vie et a mis en exergue la centralité de l'enracinement et de la tradition comme valeurs positives pour la recomposition sociétale postsocialiste. Ce point de vue qui sert la critique du socialisme d'État en avançant notamment l'idée que le régime aurait corrompu moralement la société tchèque n'est pas resté limité au monde associatif et politique. Il a en effet trouvé un relais dans la communauté des historiens en 2006 sous la plume de l'historien américain Eagle Glassheim qui oppose dans un article publié dans le

10 Christiane Brenner, "Zwischen Ost und West", Tschechische politische Diskurse 1945-1948, Munich, Oldenbourg, 2009.

11 Notes d'entretien réalisé avec l'ancien président d'Antikomplex, Ondřej Matějka, juillet 2012.

12 L'exposition Zmizelé Sudety présentée au début des années 2000 en République tchèque et en Allemagne a donné lieu à une publication en 2006 (Antikomplex et al., Zmizelé Sudety / Das Verschwundene Sudetenland, ČESKÝ LES, 2006) qui a rencontré un franc succès dans les deux pays. 
Journal of Modern History ${ }^{(14)}$ l'ordre moral harmonieux qui régnait dans les espaces frontaliers lorsqu'ils étaient habités en majorité d'Allemands avant la Seconde Guerre mondiale à l'œuvre du socialisme d'État responsable d'une part, du déracinement persistant des populations venues remplacer les Allemands et d'autre part, de la destruction à la fois des traditions locales et régionales ainsi que de l'environnement naturel des régions frontalières tchèques.

Ce point de vue est au centre des discours sur la revalorisation de l'héritage allemand en République tchèque. Il est au diapason des discours sur la Heimat des associations sudètes qui associent la fonction morale du rapport social à l'espace à l'idée d'une société enracinée dans un espace identitaire baigné de tradition chrétienne. L'association sudète catholique Ackermann Gemeinde (par ailleurs partenaire principal de l'association Antikomplex) identifie ainsi son existence à un poème en prose (Der Ackermann von Böhmen/Le laboureur de Bohême) écrit au début du XV siècle par Johannes von Saaz dont l'œuvre symbolise, pour les associations sudètes en Allemagne, les racines de la présence allemande en Bohême, la créativité de la culture de cette société et le modèle d'une vie sociale fondée sur la foi chrétienne ${ }^{(15)}$.

Ces énoncés réactualisés en République tchèque se situent dans la lignée de la pensée völkisch issue de la révolution conservatrice du XIX ${ }^{\mathrm{e}}$ siècle qui place l'ethnie (le Volk) et l'origine, localisée dans un sol (Boden), au centre de la définition de la nation ${ }^{(16)}$. Dans le contexte tchèque, ces discours magnifient l'ancienneté du peuplement allemand, l'ordre ancestral des sociétés rurales allemandes dans les pays tchèques, le rapport à l'environnement naturel symbolisé par une forme particulière de traditions historiques et de paysage culturel (Antikomplex promeut l'existence d'un « paysage culturel sudète $\left.{ }^{(17)}\right)$. C’est dans la perspective d'une réévaluation de l'héritage des populations transférées que s'inscrit le projet d'exposition permanente des Allemands des pays tchèques dans le musée d'Ústí nad Labem. Mais avant d'entrer dans l'exposé des cadres cognitifs de cette exposition, il convient de fournir quelques éléments de compréhension du contexte des musées régionaux dans lequel ce projet s’inscrit.

14 Eagle Glassheim, «Ethnic Cleansing, Communism, and Environmental Devastation in Czechoslovakia’s Borderlands, 1945-1989 ", Journal of Modern History, 78/1 (2006), p. 65-92. Cet article a également été publié dans l'une des principales revues d'histoire contemporaine tchèque sous le titre "Etnické čistky, komunismus a devastace životního prostředí: Vytváření nové identity severočeského pohraničí (1945-1989) », Soudobé dějiny, 12/3-4 (2005), p. 432-464.

15 Idée formulée sur le site Internet de l'association: www.ackermann-gemeinde.de/ueber-uns/zurgeschichte.html: "Diese bedeutende Prosadichtung aus dem Jahre 1400 ist ein Dokument für die Jahrhunderte alte Verwurzelung und schöpferische Kraft der deutschen Kultur in den Böhmischen Ländern und ein Sinnbild für eine Lebensgestaltung aus christlichem Glauben und Hoffen. »

16 George L. Mosse, Les racines intellectuelles du Troisième Reich. La crise de l'idéologie allemande, Paris, Calmann-Lévy, 2006; Tobias Weger, "Die 'Volksgruppe' im Exil? Sudetendeutsche Politik nach 1945 », in: Hans Henning HAHN (dir.), Hundert Jahre sudetendeutsche Geschichte. Eine völkische Bewegung in drei Staaten, Francfort-sur-le-Main, Peter Lang (Die Deutschen und das östliche Europa), 2007, p. 277-301; Samuel SALzBorn, «Ein Jahrhundert sudetendeutsche völkische Bewegung. Politikwissenschaftliche Dimension eines zeitgeschichtlichen Forschungsgegenstandes », in: H.H. Hahn (dir.), ibid., p. 303-322.

17 Antikomplex et al., Zmizelé Sudety (note 12). 


\section{Représenter les Allemands dans les musées régionaux sous le socialisme d'État}

Afin d'analyser la gestion muséale du passé allemand durant la période socialiste, nous rappellerons quelques éléments de l'histoire des musées régionaux à partir de l'exemple du musée de Liberec (en allemand Reichenberg, ville située au nord de la Bohême à la frontière polono-allemande). Il ne s'agit pas de faire du cas de Liberec un exemple représentatif de l'évolution générale de la situation politique et identitaire des Allemands tchèques jusqu'en 1945 mais plutôt de considérer Liberec en tant qu'« exemplar» (à la manière de Thomas $\mathrm{Kuhn}^{(18)}$ ) à partir duquel on appréciera la transformation sociétale post-1945 et la gestion du passé des sociétés allemandes durant la période socialiste. Du point de vue de leurs fonctions politique et identitaire, les musées régionaux des autres villes des régions de Bohême à majorité allemande (Cheb/Eger, Ústí nad Labem/Aussig, Děčin/Tetschen, Frýdlant/Friedland) connurent, de la fin du XIX ${ }^{\mathrm{e}}$ siècle à la chute des régimes socialistes, une évolution comparable.

Peuplée à majorité d'Allemands avant 1945, Liberec était une ville de référence de la germanité sudète. De la fin du XIX ${ }^{e}$ siècle à la création de la Première République tchécoslovaque en 1918, l'élite allemande s'est efforcée d'ériger la ville en capitale des Allemands des pays tchèques et la ville figura durant l'entre-deux-guerres à l'avant-poste de la contestation allemande vis-à-vis de la politique des nationalités de la République tchécoslovaque $^{(19)}$. Son élite politique et intellectuelle était très largement favorable à un rattachement à l'Allemagne. Politiquement, elle était à la fois l'un des haut-lieux du mouvement ouvrier (Deutsche sozialdemokratische Arbeiterpartei in der Tschechoslowakischen Republik - DSAP créé en 1919 à Teplice/Teplitz) et du parti nationaliste sudète pronazi dirigé par Konrad Henlein (créé en 1935 à Liberec sous l'appellation Sudetendeutschepartei - SdP).

D'un point de vue identitaire, Liberec est également une référence. Le romancier nationaliste sudéto-allemand, Friedrich Jaksch, y créa en 1923 la bibliothèque des Allemands de Tchécoslovaquie (Bücherei der Deutschen in der Tschechoslowakei), outil symbolique de propagande censé représenter par sa collection de la production littéraire la supériorité intellectuelle des Allemands sur les Tchèques. C'est à Liberec qu'est fondée en 1904 la Société scientifique allemande (Deutsche wissenschaftliche Gesellschaft) qui deviendra en 1924 l'institution centrale de recherche sur l'ethnographie sudète, sous l'impulsion d'Erich Gierach (philologue de l'Université allemande de Prague et membre du SdP puis du NSDAP en 1938). Les travaux d'ethnographie parrainés par cette société savante et produits dans le cadre d'une coopération scientifique avec l'Université allemande de Prague ont servi l'idéologie du mouvement völkisch sudète dans le cadre du Volkstumkampf (le combat pour la reconnaissance de l'identité du Volk allemand et de sa supériorité en Europe) ${ }^{(20)}$. En 1938, Liberec devient

18 Thomas KuHn, The structure of scientific revolution, University of Chicago press, 2012 (4e éd.).

19 Miloslava Melanová, " Hlavní město německých Čech? Liberec 1848-1918 " (La capitale des Allemands de Bohême? Liberec 1848-1918), in: Krístina Kaiserová \& Miroslav Kunštat (dir.), Hledání centra. Vědecké a vzdělávací instituce Němců v Čechách v 19. a prvnípolovině 20. století (À la recherche des centres. Les institutions scientifiques et éducatives des Allemands des pays tchèques au XIX ${ }^{\mathrm{e}}$ siècle et durant la première moitié du Xx ${ }^{\mathrm{e}}$ siècle), Ústí nad Labem, Albis, 2011, p. 300.

20 Petr Lozoviuk, Interethnik im Wissenschaftsprozess - Deutschsprachige Volkskunde in Böhmen und ihre gesellschaftlichen Auswirkungen, Leipziger Universitätsverlag, 2008, p. 250. 
le chef-lieu du Sudetengau - l'unité administrative du III ${ }^{\mathrm{e}}$ Reich unissant les régions tchèques majoritairement peuplées d'Allemands.

Créé en 1873 en tant que musée des arts décoratifs, le musée de Liberec devint, dans les années 1920, l'un des centres de production et de diffusion des savoirs valorisant la richesse culturelle et économique des «Allemands sudètes ». À la suite des migrations forcées, l'État tchécoslovaque hérita des fonds bibliographiques et muséaux. Le musée devint alors un des lieux où l'on donne à voir le projet d'une nouvelle société énoncé dans les discours sur "l'ère des bâtisseurs". Ce leitmotiv présent dans les principales villes des régions frontalières reste présent après 1948 avec la mise en place d'une censure des productions muséales (notamment les expositions, les catalogues d'expositions et les bulletins des activités des musées). Lors d'un entretien, un conservateur du musée de Liberec rappelle la manière dont le filtre idéologique de la période socialiste s'appliquait à l'exposition consacrée à l'histoire de la région et à sa dimension allemande:

«En 1983, nous avons voulu réaliser une exposition sur le mouvement architectural de la Sécession en Bohême du Nord d'un point de vue artistique et historique. L'exposition a eu lieu et elle présentait la part des Tchèques dans le mouvement architectural du début $\mathrm{XX}^{\mathrm{e}}$ siècle. Mais en réalité, l'influence allemande était très forte sur ce mouvement architectural. Elle ne devait toutefois pas être soulignée. Nous avions préparé un catalogue de l'exposition qui n'a pas été rendu public car il comportait trop de noms allemands parmi les architectes des bâtiments les plus célèbres de la ville.

Présenter la ville de Liberec comme un lieu central de l'histoire du XIX ${ }^{e}$ et du $\mathrm{XX}^{\mathrm{e}}$ siècle, était totalement impensable. Au mieux, nous pouvions traiter du mouvement ouvrier et de la social-démocratie... mais là encore, il ne fallait pas mentionner le fait que le parti communiste allemand tchécoslovaque avait été fondé avant le parti communiste tchèque. En raison de la pression idéologique, personne ne souhaitait engager des recherches sur le passé récent, et puis, pourquoi l'aurions-nous fait, la direction du musée surveillait étroitement nos activités. Faire une exposition centrée sur Liberec était de ce fait extrêmement compliqué. Nous pouvions exposer l'histoire plus ancienne, le XVIII ${ }^{\mathrm{e}}$ siècle par exemple, le début de l'industrialisation, etc. Après 1990, nous nous sommes dit qu'il fallait faire connaître le passé récent de la ville et présenter l'importance de sa place dans l'histoire du XXe siècle. C'est la raison pour laquelle, entre 1990 et 2000, nous avons présenté cinq expositions sur "Liberec, ville du $\mathrm{XX}^{\mathrm{e}}$ siècle". C'était nouveau et il m'a semblé alors qu'il y avait toujours eu une grande demande pour ce type de présentation du passé »(21).

L'histoire régionale est empreinte de la présence allemande et celle-ci a fait l'objet jusqu'en 1989 d'une censure à la fois chronologique (exclusion du $\mathrm{XX}^{\mathrm{e}}$ siècle) et thématique, portant sur les phénomènes sociaux, politiques et culturels. Ce contrôle par la censure et les réflexes d'autocensure qu'ils ont induits limitaient considérablement l'activité des musées municipaux et régionaux et faisaient peser de lourdes contraintes sur le travail des conservateurs lorsqu'ils abordaient les phénomènes culturels et les événements politiques les plus significatifs de l'histoire contemporaine des pays tchèques. D'un point de vue scientifique, ces institutions ont poursuivi durant la période socialiste la tradition ethnographique développée au cours du XIX siècle dans le cadre de la Heimatkunde (dans les régions où la majorité de la population était

21 Notes d'entretien réalisé avec un conservateur du musée de Liberec, juillet 2012. 
de langue allemande) ou de la Vlastivěda (dans les régions majoritairement tchécophones). Sans entrer dans les détails de ces cadres de production du savoir ethnographique, rappelons que chacune des traditions scientifiques s'est efforcée d'alimenter et de justifier le sentiment national des communautés linguistiques des pays tchèques en plaçant l'ethnicité et le lien à l'espace de vie local et/ou régional au centre de la définition identitaire des groupes nationaux. Dans les régions, les musées étaient les relais locaux de la construction des sociétés nationales et ont accompagné et justifié les dérives racialistes des politiques nationalitaires des années 1930. Durant toute la première moitié du $\mathrm{XX}^{\mathrm{e}}$ siècle, les musées et les sociétés savantes des grandes villes peuplées majoritairement d'Allemands (Cheb, Ústí nad Labem, Liberec) ont ainsi joué un rôle central dans le glissement d'une pratique ethnographique centrée de prime abord sur les lieux (Heimatkunde), vers une science centrée sur la description des caractéristiques des communautés locales et régionales (Volkskunde) qui, par le biais de «l'éducation du peuple» (Volksbildung) et de la propagande du parti pronazi SdP, produisit une identité nationale sudète réunissant l'ensemble des Allemands tchèques ${ }^{(22)}$.

\section{L'exposition permanente d'Ústí nad Labem: refonder le sens de l'histoire tchèque par la langue et la géographie}

L'ouverture après 1989 de débats sur le passé allemand à Ústí nad Labem est l'œuvre d'un groupe d'historiens, d'archivistes et conservateurs locaux, formés durant la période socialiste, qui ont accompagné la création en 1991 de l’Université J. E. Purkyně à Ústí nad Labem où a été créé le département d'études slaves et germaniques. Afin d'attirer l'attention du public local sur le passé allemand de la ville et de sa région environnante, les membres du département créent tout d'abord la Société pour l'histoire des Allemands en Bohême (Společnost pro dějiny Němců v Čechách), établie sur le modèle des sociétés savantes du XIX ${ }^{\mathrm{e}}$ siècle. La société finance la maison d'édition Albis qui publie, depuis le début des années 1990, les travaux de ses membres et des spécialistes tchèques du passé allemand dans les pays tchèques. C’est au sein de ce noyau de spécialistes de l'histoire locale et à la faveur de l'évolution des représentations des Tchèques vis-à-vis de la présence passée des Allemands (notamment à travers la visibilité grandissante du discours d'Antikomplex) qu'en 2006 germe l'idée de fonder le Collegium Bohemicum - un centre de recherche hébergé par le Musée municipal de la ville d'Ústí nad Labem dont l'objectif principal est la réalisation de «l'Exposition permanente de l'histoire et de la culture des habitants germanophones des pays tchèques ». Lors de sa création, le projet bénéficie du soutien des responsables municipaux dont l'ancien maire Petr Gandalovič, personnalité politique de premier plan du parti conservateur-libéral ODS dont il a été l'un des membres fondateurs au début des années 1990.

Financièrement, le projet du centre de recherche dispose dès sa création de fonds venus de l’Allemagne par la voie de la Fondation Robert-Bosch qui soutient le musée

22 Miroslav KunšTat, «Centra a periférie českých Němců ve vědě a vzdělávání » (Centres et périphéries de la science et de l'éducation des Allemands de Bohême), in: Kaiserová/Kunštat (dir.), Hledání centra (note 19), p. 40. Voir dans le même ouvrage, Petr Lozoviuk, "Etnografie jako národní věda » (L'ethnographie comme science nationale), p. 59-98. 
municipal et l'aide à pérenniser ses activités. Les travaux du Collegium sont suivis par un conseil scientifique où siègent de manière paritaire des spécialistes tchèques et allemands. Ce conseil rassemble des personnalités dont les travaux s'inscrivent dans des approches et des traditions scientifiques différentes pour ne pas dire opposées. On trouve ainsi en son sein Peter Becher, le président de l'association sudète Adalbert-Stifter-Verein (fondée à Munich en 1947) et des historiens comme Peter Haslinger (le directeur de l'Institut Herder de Marburg) dont les travaux s'inscrivent dans le renouvellement de la Ostforschung. Néanmoins, le conseil regroupe également des personnalités comme l'historien Tobias Weger (Institut pour l'histoire et la culture de l'Est européen - BKGE) dont les analyses critiquent le maintien des discours d'inspiration völkisch des associations sudètes. On compte également l'historien Martin Schulze Wessel, le directeur du Collegium Carolinum (Institut de recherche spécialisé dans l'histoire des pays tchèques, fondé en 1956 à Munich et considéré par les spécialistes comme l'héritier des départements des sciences humaines et sociales de l'université allemande de Prague). Du côté tchèque, le conseil rassemble les principaux spécialistes des relations germano-tchèques et des acteurs de premier plan du dialogue sur l'histoire commune des deux pays et du débat sur le sens de l'histoire tchèque, comme par exemple l'historien et politiste Miroslav Kunštát, l'historien Jiří Pešek et le sociologue Miloš Havelka. Le politologue français et ancien conseiller de Václav Havel, Jacques Rupnik, est également un membre actif du conseil scientifique. La présence de personnalités aux orientations politiques et scientifiques différentes, voire opposées, témoigne de la volonté de consensus qui préside au projet d'exposition et donc du caractère éminemment politique de la gestion de l'héritage des Allemands tchèques.

Le choix de définir les anciens habitants allemands du pays par la pratique de la langue allemande révèle l'ancrage politique et scientifique que les auteurs ont voulu donner à l'exposition. Le porte-parole du Collegium Bohemicum commente ainsi le titre de l'exposition:

"Si nous avions seulement dit "Allemands" nous aurions omis une grande partie de la population, les juifs par exemple. On ne peut pas dire qu'ils étaient de nationalité tchèque ou allemande, puisqu'une grande partie d'entre eux se disait de nationalité juive, mais ils parlaient allemand. Concernant les Allemands déplacés, ceux que l'on appelle les "Allemands sudètes", lorsqu'on leur demande s'ils sont allemands, ils répondent par exemple: "non, je suis un Alt-Österreicher" (un Allemand de l'Autriche-Hongrie). Et puis, il faudrait encore faire la différence entre les Allemands qui vivaient en Tchécoslovaquie et les Allemands dits de l'Empire. Ce qui pose d'autres types de problèmes. Tout cela montre qu'en tchèque nous n'avons pas suffisamment de mots pour désigner ces différentes catégories de population. En nous appuyant sur la langue et non sur la nationalité, nous nous plaçons dans une démarche inclusive »(23).

En proposant de s'appuyer sur la langue comme critère de définition des populations dont on souhaite exposer l'histoire et l'apport culturel, les responsables du Collegium Bohemicum entendent se distancier de l'approche völkisch ${ }^{(24)}$ qui définit la nationalité allemande des populations déplacées de l'Europe centrale et de l'Europe de l'Est vers

23 Notes d'entretien, secrétaire général du Collegium Bohemicum, octobre 2012, Ústí nad Labem.

24 G.L. Mosse, Les racines intellectuelles du Troisième Reich (note 16). 
l'Allemagne à partir du concept racial de Volk et de Stamm (littéralement, la tribu ${ }^{(25)}$ ). Le Collegium affiche son rejet des catégories raciales de Volksdeutsche (les Allemands ethniques et non les citoyens de l'Allemagne) et de Reichsdeustche (Allemands ethniques citoyens de la République de Weimar puis du III ${ }^{e}$ Reich) dont la propagande nazie avait fait l'un des instruments du discours de supériorité raciale légitimant la conquête des territoires de l'Est de l'Europe et dont les associations sudètes font encore usage pour distinguer leur identité de celle des Allemands non originaires de l'Europe centrale et orientale ${ }^{(26)}$.

La mobilisation du critère linguistique rappelle les termes d'un débat sur les principes du programme national tchèque qui avait opposé au début du XIX ${ }^{\mathrm{e}}$ siècle l'écrivain Josef Jungmann (1773-1847) au mathématicien et philosophe Bernard Bolzano (1781-1848). Alors que Jungmann définissait le programme du renouveau national à partir de la théorie herdérienne de la nation linguistique ${ }^{(27)}$, Bolzano lui opposait l'idée d'une communauté nationale fondée sur le principe du patriotisme territorial ${ }^{(28)}$. Deux propositions opposées que, plus d'un siècle plus tard, le philosophe Jan Patočka (1907-1977), l'un des fondateurs de la dissidence politique tchèque des années 1970, s'est efforcé de relier dans son essai de philosophie de l'histoire, Qu'est-ce que les Tchèques? ${ }^{(29)}$.

Sur la question linguistique, les responsables du Collegium renouent avec les enjeux nationalitaires qui avaient opposé nationalistes tchèques et allemands sur les usages de la langue comme critère de définition statistique des communautés nationales sous la monarchie habsbourgeoise et durant la Première République tchécoslovaque. Devait-on choisir le critère restreint de la langue maternelle soutenu par les Tchèques (de la même manière que dans les territoires administrés par la Couronne de Hongrie) afin de réduire le nombre des ressortissants Allemands? Ou devait-on au contraire mesurer le nombre de ressortissants d'une nationalité à partir de la langue d'usage, comme le souhaitaient les Allemands afin de comptabiliser parmi les Allemands de nombreux individus pratiquant indifféremment les deux langues dans la vie quotidienne? Pour définir les populations présentées dans l'exposition, les membres du Collegium adoptent ce dernier point de vue afin d'inclure toutes les personnes qui, par le passé, mobilisaient l'allemand dans leur vie privée et/ou publique. Ce parti pris permet également de rappeler la présence des quelques milliers d'Allemands qui ont pu rester à la suite des migrations forcées de population mais auxquels peu de travaux ont

25 Tobias Weger, "Volkstumskampf” ohne Ende? Sudetendeutsche Organisationen 1945 bis 1955, Francfort-sur-le-Main, Peter Lang (Die Deutschen und das östliche Europa, 2), 2008.

26 Doris L. Bergen, "The Nazi Concept of 'Volksdeutsche' and the Exacerbation of Anti-Semitism in Eastern Europe, 1939-1945 », Journal of Contemporary History, 29/4 (1994), p. 569-582.

27 Josef Jungmann, «Dvojí rozmlouvání O jazyku českém » (Deux dialogues sur la langue tchèque), Hlasatel česky, 1806.

28 Bernard Bolzano, O poměru obou národností v Čechách (Sur la relation des deux nationalités en Bohême), 1816.

29 Jan Patočka, Co jsou Češi? (Qu'est-ce que les Tchèques ?), Prague, Panorama, 1992; du même auteur: «Dilema v našem národním programu - Jungmann a Bolzano » (Les dilemmes de notre programme national: Jungmann et Bolzano), in: Jan PAтočKA, Náš národní program (Notre programme national), Prague, Evropský kulturní klub, 1990, p. 41-56. 
été consacrés ${ }^{(30)}$. Ce choix permet en outre de présenter une réalité sociale complexe qui a traversé l'histoire des pays tchèques et dont ne peut rendre compte l'utilisation des critères retenus dans le discours völkisch qui distingue les membres des nationalités à partir d'une ethnicité considérée comme objective.

Pour présenter, en parallèle du principe linguistique (Jungmann), l'idée d'un patriotisme territorial (Bolzano) permettant de transcender les clivages nationaux, les responsables du Collegium s'appuient sur des références aisément identifiables par les visiteurs; ainsi le choix de présenter l'entreprise de production d'eau minérale Mattoni, l'une des plus connues de République tchèque:

«Un bel exemple est celui de Mattoni. Lorsque l'on évoque Mattoni, tout le monde pense à la République tchèque, c'est d'ailleurs l'eau de nombreuses manifestations officielles. Nous présentons ainsi le cas de Mattoni et interrogeons la sonorité du nom. Mattoni ne sonne ni tchèque ni allemand, mais plutôt italien pour la bonne raison que la famille de l'entrepreneur venait d'Italie. Or les membres de la famille Mattoni se sont installés dans les régions frontalières et s'exprimaient en allemand [...]. Si on avait interrogé le dernier descendant de la famille avant la Seconde Guerre mondiale pour savoir s'il était tchèque ou allemand, il vous aurait sans doute répondu: Ich bin aus Böhmen (je viens de Bohême). Cette conception de l'identité liée au Vaterland et à la Heimat correspond à un patriotisme territorial qui est toujours présent chez les Allemands expulsés " ${ }^{(31)}$.

De prime abord, la référence au territoire de la Bohême (Böhmen) renvoie ici au problème bien connu de la non-coïncidence entre les langues tchèque et allemande. L'allemand permet de distinguer des réalités différentes à partir des adjectifs böhmisch («bohêmien » qui renvoie au territoire), deutsch et tschechisch (allemand et tchèque qui renvoient à la langue et à la nationalité). La langue tchèque ne permet pas de distinguer deux réalités distinctes puisque l'adjectif český (tchèque) renvoie à la fois à la langue, au national et au territoire.

Dans la perspective de la synthèse entre identité linguistique et identité territoriale proposée par Patočka, les membres du Collegium ont recours à l'espace identitaire de la Bohême. Cette référence permettrait d'accéder à la compréhension de l'évolution du contexte social et culturel au cours de l'histoire des pays tchèques en évacuant le cadre ethno-national. Cette démarche suit les propositions de la nouvelle historiographie américaine qui présente les conflits communautaires entre les Tchèques et les Allemands (de 1848 à 1948) comme le fait de groupes politiques nationalistes dont les modes de mobilisation des masses et les cadres de pensée étaient à la fois similaires et concurrents ${ }^{(32)}$. Cette historiographie est indéniablement influencée par les thèses

30 Olga Šmidova, "Dans l'ombre des Sudètes, mémoire et identité collective des Allemands tchèques ", Revue d'études comparatives Est-Ouest, 41/1 (2010), p. 141-162.

31 Note d'entretien, secrétaire général du Collegium Bohemicum (note 23).

32 On se référera à la nouvelle génération d'historiens spécialistes du nationalisme en Europe centrale tels que Jeremy King et Tara Zahra dont les travaux font actuellement référence. Les travaux de Pieter Judson portant sur le nationalisme allemand dans les territoires de l'Autriche-Hongrie (cf. Pieter Judson, Guardians of the Nation: Activists on the Language Frontiers of Imperial Austria, Harvard University Press, 2006) constituent un point d'entrée central pour saisir cette approche par le territoire du vécu des sociétés d'Europe centrale. 
du philosophe et anthropologue d'origine tchèque Ernest Gellner, selon lequel c'est le nationalisme qui crée les nations et non l'inverse ${ }^{(33)}$.

Dans ces travaux récents, les mouvements nationalistes sont présentés de manière distincte de la vie quotidienne multiculturelle des pays tchèques où la référence aux lieux de vie et aux territoires importait davantage que l'appartenance à une communauté nationale. Selon ce point de vue, jusqu'à une époque récente, les populations n’auraient été ni tchèques, ni allemandes mais "bohêmiennes ». Les individus ne se seraient pas définis par leur appartenance nationale mais par leur lieu de vie ${ }^{(34)}$.

Cet angle d'approche du passé culturel et social des pays tchèques par le territoire rejoint, mais sans se confondre totalement avec elles, les narrations identitaires des associations sudètes pour qui la Heimat (entendue ici comme le lieu d'origine) constitue la référence centrale de l'identité collective. Cette idée fut capitale dans les discours identitaires des mouvements politiques des Allemands sudètes de l'entredeux-guerres. Elle fut réintroduite dans l'immédiat après-guerre par Franz Haibach (1899-1958), l'un des fondateurs de l'Ackermann Gemeinde qui s'est appuyé sur l'identité territoriale des Allemands sudètes pour formuler les revendications du droit au retour (Heimatrecht) ${ }^{(35)}$ et a ainsi contribué au maintien après 1945 de la qualification d'un groupe ethnique (Volksgruppe) dit « sudète » et à sa reconnaissance comme tel par le gouvernement de Bavière en 1962.

La différence entre les deux formes de recours à l'espace réside, pour les tenants $\mathrm{du}$ "bohêmisme " (revendiqué par le Collegium), dans son caractère multiculturel, tandis que l'espace revendiqué de la Heimat des Allemands sudètes est pensé en fonction de l'ethnicité du groupe qu'il identifie. Ces deux propositions, multiculturelle et ethnique, ne s'opposent toutefois pas dans leur pratique politique. Ici encore, les responsables de l'Ackermann Gemeinde fournissent un exemple particulièrement intéressant de ce double usage du concept d'identité territoriale multiculturelle et du maintien de la pensée völkisch dans les discours attachés à la variante " bohêmiste ». Alors que l'Ackermann Gemeinde entretient des relations étroites avec les membres de la Fondation Bolzano qui œuvre en faveur de la primauté d'un patriotisme territorial multiculturel dans les pays tchèques, son action au sein de la Sudetenlandsmannschaft rappelle que, loin de renier la pensée völkisch, l'Ackermann Gemeinde contribue à sa diffusion dans les pays tchèques en agrégeant le principe d'une ethnicité sudète à celui du multiculturalisme.

En situant le projet d'exposition dans le cadre de la synthèse proposée par Jan Patočka, les responsables du Collegium entendent éviter l'ethnicisation de la société

33 Ernest Gellner, Saint of the Atlas, University of Chicago Press, 1969, p. 147-178. Voir chez le même auteur Nation et nationalisme, Paris, Payot, 1989, p. 76 et p. 86.

34 Tara Zahra, Pieter M. Judson, «Introduction: Sites of Indifference to Nationhood », Austrian History Yearbook, 43 (2012), p. 21-27. Voir également, Tara ZAHr A, «Imagined Noncommunities: National Indifference as a Category of Analysis », Slavic Review, 69/1 (2010), p. 93-119.

35 Pour un aperçu de l'histoire de l'Ackermann Gemeinde et de son rôle dans la construction de la germanité sudète dans l'après-Seconde Guerre mondiale, voir T. WEGER, "Volkstumskampf” ohne Ende? (note 25); Erik Franzen, Der vierte Stamm Bayerns: die Schirmherrschaft über die Sudetendeutschen, Munich, Oldenbourg, 2010. 
dont ils exposeront l'héritage et dont le discours sur le multiculturalisme est l'une des notions centrales.

Le discours sur la localisation de l'exposition dans la ville d'Ústí nad Labem participe de cette démarche. Selon les mots du directeur du Musée municipal, l'histoire de la ville est l'une des preuves de l'expérience du multiculturalisme non-national et non-ethnique:

«Les deux nationalismes, tchèque et allemand, étaient présents dans la ville depuis 1848. À côté de ces nationalismes, il y avait également une forme plus conservatrice, qui s'appuyait sur une identité territoriale de la Bohême et que représentaient l'aristocratie et les grands entrepreneurs. Ces derniers ont donné à la ville son caractère cosmopolite. Bien entendu, la majorité des habitants de la ville se déclaraient allemands lors des recensements, mais en même temps, les nationalistes allemands ne considéraient pas la ville comme allemande... à la façon de Liberec ou de Cheb [...]. La vie de l'entrepreneur libéral, Karl Wolfrum, qui possédait en 1848 une manufacture textile et une tannerie, est une belle illustration de cette idée. Lorsque la révolution a éclaté en Autriche, il a réussi à maintenir le niveau de ses affaires avec les différentes fractions qui s'affrontaient. Il a dû traverser les pays tchèques en partant d'Ústí et il est passé à Prague, puis à Brno jusqu'à Vienne. [...]. Il portait les cocardes des différents groupes qui s'affrontaient. Et, selon les clients, il arborait telle ou telle cocarde. Cette manière de faire était très courante parmi l'élite d'Ústí. C'était une stratégie pragmatique! » ${ }^{(36)}$

La narration renvoie aux manières dont les élites se sont positionnées vis-à-vis des discours nationalistes et montre que le choix de l'appartenance à la nationalité relevait plus de l'opportunisme que de la conviction, plus de la nécessité contingente que du sentiment d'appartenance. Présentée ainsi, la ville d'Ústí nad Labem justifie pleinement sa fonction de lieu privilégié pour l'exposition d'une narration historique conçue comme alternative aux grands récits nationaux.

Le porte-parole du Collegium présente ainsi le récit que les visiteurs seront prochainement invités à suivre:

«D’un point de vue géographique, nous présenterons l'ensemble des pays tchèques, et aussi les Allemands des Carpates qui étaient présents durant la Première République en Slovaquie et en Ruthénie subcarpatique. D’un point de vue chronologique, l'exposition commence avec ce que l'on appelle la deuxième colonisation des pays tchèques au cours du XIII ${ }^{\mathrm{e}}$ siècle jusqu'en 1947, lorsque s'arrête officiellement les transferts des Allemands.

Dans le contenu, nous souhaitons utiliser certains matériaux, tels que les robes, les costumes folkloriques par exemple pour montrer que la construction nationale qui affirme que les Allemands sudètes sont une nation, n'a pas de sens, car du point de vue de la langue comme de celui du développement identitaire, il s'agissait de groupes distincts qui n'avaient pas grand-chose en commun, si ce n'est des langues dialectales partageant la même racine allemande. Nous montrons ainsi qu'à l'échelle locale et régionale, les Allemands de Cheb (à l'extrême ouest de la Bohême) étaient plus proches des Tchèques qui vivaient à quelques kilomètres de là, que des Allemands d'Opava (au nord-est de la Moravie). Nous recevons de nombreuses critiques disant: “vous racontez le passé à votre manière”. Nous souhaitons au contraire présenter les objets du passé que nous réunissons avec les points de vue de l'époque. Car nous souhaitons donner au visiteur les moyens de se faire une opinion. Nous fournissons seulement des indices pour que le visiteur se représente la manière dont les

36 Notes d'entretien, directeur du musée municipal d’Ústí nad Labem, octobre 2012. 
choses étaient perçues après 1945, comment l'historiographie marxiste s'est emparée de la question allemande, comment les Allemands ont considéré leur histoire, comment 1989 a apporté de nouveaux éléments, etc. ${ }^{(37)}$.

L'exposition propose une réflexion sur le sens à donner aux traces du passé et à l'assignation de valeurs pour l'histoire tchèque. Les responsables du Collegium inscrivent la narration de l'exposition dans les pas du premier président de la Tchécoslovaquie et père spirituel du tchécoslovaquisme, Tomas Garrigue Masaryk, qui désigna, dans sa déclaration à l'Assemblée nationale du 22 décembre 1918, les Allemands tchécoslovaques par l'expression "nos Allemands » (naši Němci) pour exprimer l'idée d'un pacte transnational regroupant l'ensemble des communautés du nouvel État. Placé sous l'égide de cette figure référentielle positive, le visiteur sera amené à se poser les questions suivantes: Qui étaient «nos Allemands »? Que sont-ils devenus? Comment trouver les traces de leur présence passée?

"Nous exposerons par exemple des objets qui ont été trouvés récemment dans le grenier d'une maison en rénovation près de Déčín (nord-ouest de la Bohême). Les Allemands avaient entreposé de nombreux biens qu'ils pensaient pouvoir récupérer. Ils croyaient qu'ils reviendraient un jour. Avec cette découverte, on voit que nous ne pouvons plus faire comme s'ils n'avaient jamais été là, comme cela a été le cas durant le communisme; ce n'est plus possible. [...] leur histoire est d'une grande importance pour notre propre histoire [...]. Qu'elles soient nationalistes, communistes ou conservatrices, les élites politiques continuent de travailler ce passé avec un fond de revanchisme. Nous sommes ainsi souvent accusés d'être la cinquième colonne car, selon eux, nous construisons un mémorial allemand ${ }^{(38)}$.

\section{Conclusion}

Dans les travaux des spécialistes des relations germano-austro-tchèques, le passé des Allemands constitue un point de repère pour la formulation de narrations sur l'identité tchèque. Ce passé est à la fois la source d'expressions négatives liées au ralliement de la grande majorité de la population allemande en faveur du rattachement des régions frontalières à l'Allemagne nazie, ou positives associées aux apports de la société allemande dans les domaines de la culture, de la politique, du développement industriel et scientifique. Occultées durant plusieurs décennies par le régime socialiste, les traces de la présence passée des Allemands tchèques sont inscrites dans une logique patrimoniale qui prend appui sur un socle de références et de concepts qui déterminent leur valorisation. La démarche muséale, liée à la tradition scientifique et éducative de la Vlastivěda, elle-même créée en référence à la Landeskunde allemande, encadre la réintroduction du passé allemand dans une nouvelle trame narrative sur l'histoire nationale tchèque. Au diapason des travaux historiographiques récents sur le nationalisme, l'initiative du Collegium Bohemicum entend dépasser la conception de l'appartenance des individus selon des catégories ethno-nationales toujours présentes dans les narrations identitaires des associations sudètes. Pour ce faire, elle assigne à la langue une importance centrale et renoue ainsi avec les enjeux linguistiques présents dans les débats sur la définition du national dans les pays tchèques dans lesquels

37 Note d'entretien avec le secrétaire général du Collegium Bohemicum (note 23). 
langues et nations se recoupent. Dans ce processus, le facteur linguistique est couplé au cadre que constitue l'espace de la Bohême. L'importance de ce dernier tient à ses diverses fonctions. Comme le montre la façon dont le directeur présente la société multiculturelle de la ville d'Ústí nad Labem, la localisation est tout d'abord un moyen efficace pour dépasser les discours nationalitaires et leur incapacité à raconter l'histoire des relations sociales dans les pays tchèques. Ce point de vue s'inscrit dans la perspective ouverte par Gellner dans laquelle les lieux jouent le rôle de référent identitaire et sont à la fois les scènes matérielles de la vie sociale et les supports de définition des sociétés ${ }^{(39)}$. L'espace est enfin une ressource symbolique pour l'élaboration d'une démarche réflexive sur le passé.

La revalorisation du passé d'avant-guerre ne s'accompagne pas de l'examen du repeuplement consécutif aux transferts. Elle évacue de la narration les trajectoires individuelles et collectives des sociétés locales qui se sont formées dans l'après-guerre et durant le socialisme. Or, c'est l'ignorance supposée de ces sociétés, conçues comme le résultat à la fois du discours nationaliste tchèque et du socialisme d'État, que le projet d'exposition entend pallier. En cela, il s'inscrit à l'échelle de l'Europe centrale et orientale dans les débats portant, plus de vingt ans après la chute du mur de Berlin, sur le caractère inachevé de la transition post-socialiste ${ }^{(40)}$. La réintroduction du passé des Allemands dans les narrations sur l'histoire nationale en République tchèque sert ainsi de repoussoir à l'expérience du socialisme d'État qu'elle entend clore en modifiant les représentations des sociétés sur leur passé.

\begin{abstract}
In 2006, the project of a permanent exhibition dedicated to the history and the cultural heritage of the German speaking Czechs have been launched in the City Museum of Usti nad Labem (North-western Bohemia). This article proposes to analyze the cognitive frame that support the narration of the exhibition's project. Particular attention is paid to the societal issues of the making of a renewed heritage in Czech Republic that includes, more than 70 years after the mass population transfers, the traces left by the former German societies.

\section{Zusammenfassung}

Im Jahre 2006 begann man im Stadtmuseum von Ústi nad Labem (Nordwest-Böhmen) mit dem Projekt einer ständigen Ausstellung, die der Geschichte und dem kulturellen Erbe der deutschsprachigen Bevölkerung in den böhmischen Ländern gewidmet werden soll. Dieser Beitrag hat zum Ziel, den kognitiven Rahmen zu analysieren, der dem Narrativ des Ausstellungsprojektes zugrunde liegt. Einen besonderen Akzent legt die Untersuchung auf die gesellschaftliche Dimension dieses erneuerten Erbes in der heutigen Tschechischen Republik sowie auf den Umgang mit den Spuren der damaligen deutschsprachigen Gesellschaft mehr als 70 Jahre nach der Zwangsumsiedlung.
\end{abstract}

39 E. Gellner, Saint of the Atlas (note 33).

40 James Mark, The Unfinished Revolution: Making Sense of the Communist Past in Central-Eastern Europe, Yale University Press, 2011. 